\title{
Rheological behavior of human blood in uniaxial extensional flow
}

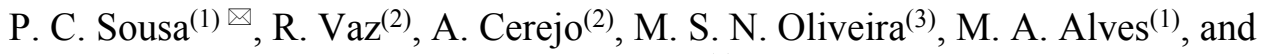 \\ F. T. Pinho ${ }^{(4)}$ \\ ${ }^{(1)}$ Departamento de Engenharia Química, CEFT, Faculdade de Engenharia da Universidade do Porto, Rua \\ Dr. Roberto Frias, 4200-465 Porto, Portugal. (psousa, mmalves@fe.up.pt) \\ ${ }^{(2)}$ Departmento de Neurocirurgia, Hospital S. João, Porto, Portugal. (ruimcvaz@gmail.com, \\ a.cerejo@sapo.pt) \\ ${ }^{(3)}$ Department of Mechanical and Aerospace Engineering, University of Strathclyde, Glasgow G1 1XJ, \\ UK (monica.oliveira@strath.ac.uk) \\ ${ }^{(4)}$ Departamento de Engenharia Mecânica, CEFT, Faculdade de Engenharia da Universidade do Porto, \\ Rua Dr. Roberto Frias, 4200-465 Porto, Portugal. (fpinho@fe.up.pt) \\ ${ }^{\circledR}$ Corresponding author: Tel: +351 225081404; Fax: +351 225081449; Email: psousa@fe.up.pt
}

\begin{abstract}
We present an investigation of the rheological behavior of whole human blood under uniaxial extensional flow. For that purpose, capillary breakup experiments were carried out by combining the slow retraction method, high-speed imaging techniques and an immiscible oil bath. The use of the oil bath was aimed at reducing liquid loss by evaporation and to reduce light refraction effects, thus allowing the visualization of the blood cells during the filament thinning. Extensional relaxation times were measured for whole blood samples collected from a total of thirteen healthy adult volunteers from both genders, with hematocrit levels between $38.7 \%$ and $46.3 \%$. For this range of red blood cell concentrations, the variation of the extensional relaxation time is small, with the average extensional relaxation times measured in air and in oil being $114 \pm 30 \mu$ s and $259 \pm 47 \mu \mathrm{s}$, respectively. An increase of the red blood cells concentration leads to an increase of the bulk viscosity of the sample, which delays the thinning of the filament and consequently the time to breakup. In addition, blood aging was found to reduce the relaxation time while the absence of anticoagulant increases it significantly.
\end{abstract}




\section{Introduction}

Blood is a multicomponent suspension exhibiting complex rheological characteristics, which shows a different behavior for blood flowing in large or small diameter vessels. The non-Newtonian behavior of blood is revealed by its shear-thinning viscosity and stress-relaxation properties. Moreover, its viscoelastic characteristics are associated with the morphological and elastic properties of the red blood cells (RBC) or erythrocytes $[1,2]$ with its elastic response being negligible under certain flow conditions. For this reason, it is expected that the viscoelasticity of blood increases with hematocrit and depends strongly on the interactions between cells. Thurston [3] was the first to recognize the viscoelastic nature of blood in shear flow. Subsequent research studies on blood viscoelasticity have focused essentially on shear flow experiments as reviewed by Sousa et al. [4], rather than on extensional rheology [5-7]. Recent experimental studies using different techniques, namely passive microrheology and large amplitude oscillatory shear measurements (LAOS), showed that blood has a small, but nonnegligible, elastic character $[7,8]$.

Microfluidics are frequently used as platforms to assess blood and RBC behavior under extensional flow $[9,10]$, since the small characteristic length scale typically allows for strong extensional flow fields to be probed in the absence of significant inertial effects. For example, the evaluation of RBC deformability in microchannels, in which a strong extensional flow is generated, is a relevant topic of research $[9,11]$ since it can be associated with the diagnosis of certain diseases. Using the cells deformability as a biophysical marker is a promising tool that can be used instead of common biochemical markers which require costly labelling and sample-preparation steps. Furthermore, generating an extensional flow field is a useful way to stretch deformable materials, such as red blood cells [12] or DNA molecules [13,14], and investigate their 
viscoelastic response under controllable flow conditions. For red blood cells, it is important to note that extensional stresses are an important factor for cell hemolysis, as demonstrated by Down et al. [15] in the flow through an axisymmetric contraction, similar to what is possibly found in blood vessels.

Extensional flow enhances viscoelastic properties and, for some complex fluids, elastic instabilities can arise due to a significant increase of the normal stresses [16-18]. Sousa et al. [10] showed that two viscoelastic blood analogue solutions with similar shear viscosities presented distinct flow behavior under a strong extensional flow in a microfluidic contraction/expansion, due to their different viscoelasticities. More recently, Brust et al. [19] found that human plasma revealed a viscoelastic behavior in a strong extensional flow, while keeping its Newtonian characteristics under a simple shear flow, and suggested that viscoelasticity may be responsible for the onset of viscoelastic instabilities especially in the presence of red blood cells at concentrations of $50 \%$. Thus, there is still a clear need to characterize blood and plasma in extensional flow in order to understand its behavior under those flow conditions. For that purpose, in this work we measure the extensional relaxation time of whole human blood using a capillary thinning device. A custom-built setup combining the slow retraction method and high-speed imaging techniques [20] is employed in order to generate a vertical stretching filament of blood. The temporal evolution of the minimum filament diameter is used, combined with an appropriate scaling law, to determine the extensional relaxation time. The measurements were performed for blood filaments surrounded by air and by an immiscible oil, which minimizes light refraction and allows the visualization of the blood cells behavior during the filament thinning and breakup. The 
effect of sample aging on the extensional flow behavior of whole human blood and of human plasma was also investigated.

\section{Experimental}

\section{A. Sample preparation}

Blood samples were collected from thirteen healthy human adult volunteers under informed consent. The donors included five female and eight male with hematocrit (Hct) ranging from $38.7 \%$ to $46.3 \%$, as described in Table I. The experimental work was carried out at Hospital de S. João (Porto, Portugal) in compliance with its Ethics Committee for Health. Blood was collected by venepuncture at the antecubital vein using $3 \mathrm{~mL}$ plastic vacuum tubes $\left(\mathrm{BD}\right.$ Vacutainers $\left.{ }^{\circledR}\right)$ containing ethylenediaminetetraacetic acid tripotassium salt $\left(\mathrm{K}_{3}\right.$ EDTA, $\left.1.8 \mathrm{mg} \mathrm{mL}^{-1}\right)$ in order to prevent coagulation [21].

After blood collection, each sample was left at room temperature during approximately 10 minutes in order to equilibrate the temperature, after which the experiment was performed. Whole blood samples were used up to a maximum of $2 \mathrm{~h}$ after blood withdrawal, unless otherwise stated.

Human plasma samples were obtained from donor D3. First, whole blood was centrifuged at a moderate speed $(2500 \mathrm{rpm})$ for 10 minutes to minimize cell damage. Then, the separated liquid phase (yellowish, top layer) was centrifuged for another 10 minutes at $2500 \mathrm{rpm}$, and the plasma was removed by aspiration. In addition, the RBC (red lower layer) obtained in the first centrifugation were washed twice with phosphate buffered saline (PBS, $\mathrm{pH}=7.4$ ) and centrifuged again at the same speed. The washed $\mathrm{RBC}$ were suspended in plasma or PBS in order to prepare samples with an hematocrit of $20 \%(v / v)$. 
TABLE I. Gender and values of hematocrit for each donor.

\begin{tabular}{ccc}
\hline Donor & $\begin{array}{c}\text { Female (F) / } \\
\text { Male (M) }\end{array}$ & Hct (\%) \\
\hline D1 & F & 38.7 \\
D2 & F & 39.2 \\
D3 & F & 40.3 \\
D4 & F & 40.8 \\
D5 & F & 42.6 \\
D6 & M & 39.8 \\
D7 & M & 40.4 \\
D8 & M & 41.6 \\
D9 & M & 42.4 \\
D10 & M & 42.4 \\
D11 & M & 45.2 \\
D12 & M & 45.8 \\
D13 & M & 46.3 \\
\hline \hline
\end{tabular}

\section{B. Experimental set-up}

Measurements of the filament thinning dynamics of the blood samples were carried out using a custom-made capillary breakup device. The experimental setup used in the investigation is described in detail in Sousa et al. [20]. The blood samples are placed between two cylindrical rods $(2 \mathrm{~mm}$ in diameter), which are initially $\sim 500 \mu \mathrm{m}$ apart. The liquid bridge confined between the circular plates is then stretched as one rod, which is mounted on a motorized stage (Zaber, Tech T-LSM100A), moves at a constant speed of $10 \mu \mathrm{m} / \mathrm{s}$. Preliminary tests were performed using horizontal stretching of blood samples, as described in Sousa et al. [20], but the results here presented were obtained using a vertical filament stretching as depicted in Fig. 1, in order to minimize the effects of sedimentation of blood cells in the region of interest where the measurements were performed, i.e. in the middle region of the filament. 


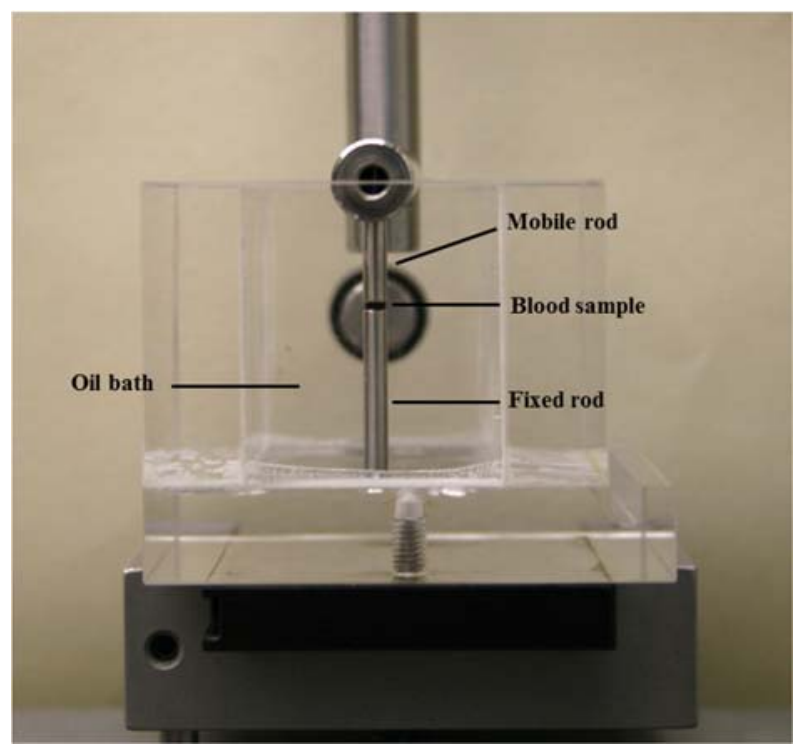

FIG. 1. Detailed view of the test cell of the experimental setup. In the experiments, the top rod moves at a constant velocity of $10 \mu \mathrm{m} / \mathrm{s}$ while the bottom rod remains stationary. The blood sample is placed in between the rods with an initial gap of about $500 \mu \mathrm{m}$. More details of the experimental setup are presented in Sousa et al. [20].

To reduce inertial effects, the slow retraction method developed by Campo-Deaño and Clasen [22] was employed, where the velocity of the movable rod was low enough to ensure that the liquid bridge undergoes a sequence of equilibrium states until the elastocapillary instability occurs spontaneously. Digital images were acquired using a highspeed CMOS camera (FASTCAM Mini UX100) coupled with a high-resolution lens system (Optem Zoom 70 XL). Since blood is a weakly elastic fluid, the frame rate used needs to be high enough to capture the relevant filament-thinning regimes, especially the exponential decay of the filament diameter. Additionally, the optical magnification must be sufficiently high to resolve the filament diameter even in the late stages near breakup. Typically, we used a spatial resolution of 1280 pixels $\times 152$ pixels, at 32768 frames per second (fps) and an exposure time of $3.9 \mu \mathrm{s}$. The resulting image scale factor was $1.16 \mu \mathrm{m} /$ pixel. The images were digitally processed to detect the filament interface 
with sub-pixel resolution $[20,23]$. The minimum diameter of the filament, along the filament axis, was determined for each acquired image. Direct measurements of the time evolution of the minimum filament diameter enabled quantitative determination of the blood extensional relaxation time $(\lambda)$.

For viscoelastic fluids, the filament thinning is initiated by a capillary instability and then generates a quasi-cylindrical filament in which elastic and capillary forces balance, whereas inertial, viscous and gravitational forces are frequently negligible [24]. Elastocapillary balance has been observed in the filament thinning of high-viscosity viscoelastic solutions [25-27] as well as low-viscosity viscoelastic solutions [22,28,29]. In the elasto-capillary regime, the filament diameter $(D)$ decays exponentially in time according to $[25,30]$ :

$$
\frac{D(t)}{D_{0}}=\left(\frac{G D_{0}}{4 \sigma}\right)^{1 / 3} \exp \left(-\frac{t}{3 \lambda}\right)
$$

where $D_{0}$ is the initial diameter, $G$ is the fluid elastic modulus, $\sigma$ is the surface tension and $t$ is the time. Equation (1) can be re-written as $D(t) / D_{0}=C \exp [-t /(3 \lambda)]$, where $C=\left(G D_{0} /(4 \sigma)\right]^{1 / 3}$ is a constant. The blood relaxation time is calculated from the slope of the linear fit to the experimental data of $\log \left[D(t) / D_{0}\right]$ as a function of time, $t$.

We investigated the elongational flow behavior of whole blood for samples surrounded by air and by an immiscible silicone oil with a kinematic viscosity of $0.65 \mathrm{cSt}$ (Xiameter PMX-200, Dow Corning). The use of an outer immiscible liquid bath, in which the liquid bridge is completely submerged, eliminates water evaporation from the solvent and allows the visualization of the blood cells in the filament because the presence of an outer fluid minimizes the refraction of light in the cylindrical filament and consequently the cells can be observed in the acquired images. We use a low 
viscosity silicone oil in order to minimize the shear at the interface between the filament and the oil, which was found by Sousa et al. [20] not to affect significantly the measurement of the extensional relaxation time.

Since the capillary thinning process of the blood samples is fast, and occurs at high strain rates, the advection of the red blood cells is fast and Brownian motion can be neglected. This can be attested using the Péclet number, $P e$, which quantifies the ratio of the rate of advection of the flow to the rate of diffusion by Brownian motion [31],

$$
P e=\frac{6 \pi \eta_{p} \dot{\varepsilon} R_{R B C}^{3}}{k_{B} T}
$$

where $k_{B}$ is the Boltzmann constant, $T$ is the absolute temperature, $\eta_{\mathrm{p}}$ is the plasma shear viscosity, $R_{\mathrm{RBC}}$ is the red blood cells equivalent radius and $\dot{\varepsilon}$ is the strain rate of the filament, which is defined as:

$$
\dot{\varepsilon}=-\frac{2}{R} \frac{\partial R}{\partial t}
$$

Considering $\eta_{p}=2 \mathrm{mPa}$ s and $R_{\mathrm{RBC}}=4 \mu \mathrm{m}$ [4], the smallest Péclet number found in the experiments is of the order $P e=\mathcal{O}\left(10^{6}\right)$, which justifies neglecting Brownian motion.

Each test was repeated at least three times to assess the repeatability and to calculate the standard deviation of the measured relaxation times. All measurements were performed at room temperature, $T=21 \pm 2{ }^{\circ} \mathrm{C}$.

\section{Results and Discussion}

\section{A. Effect of sedimentation of the blood cells}

Preliminary tests were performed with the blood filament placed in the horizontal position, as in the experiments of Sousa et al. [20]. The visualization of the filament 
dynamics showed the presence of a plasma-rich layer on the filament top edge between the surrounding medium and blood due to sedimentation of the blood cells. Consequently, the concentration of cells in the cylindrical thread during the slow retraction was changing in time. In order to minimize the effect of cell sedimentation, we performed experiments with vertically stretched blood filaments. The capillary bridge formed between the two rods is stretched as the top plate moves linearly at slow velocity. Visualizations of the entire filament thinning process, in the vertical direction, showed that a small plasma layer still forms during the experiment but is localized near the top plate rather than in the central region of the filament, as was the case for the horizontal threads, therefore not affecting the concentration of cells in the measurement region of minimum filament diameter. Figure 2 compares the diameter evolution of the blood filament thinning horizontally and vertically when surrounded by air (Fig. 2a) and by oil (Fig. 2b).

(a)

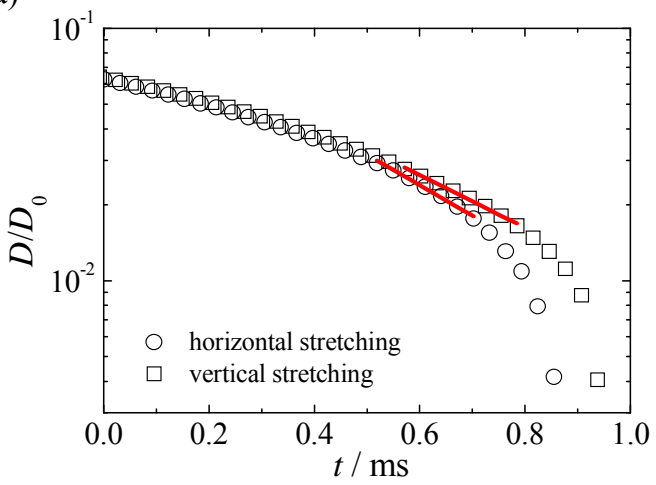

(b)

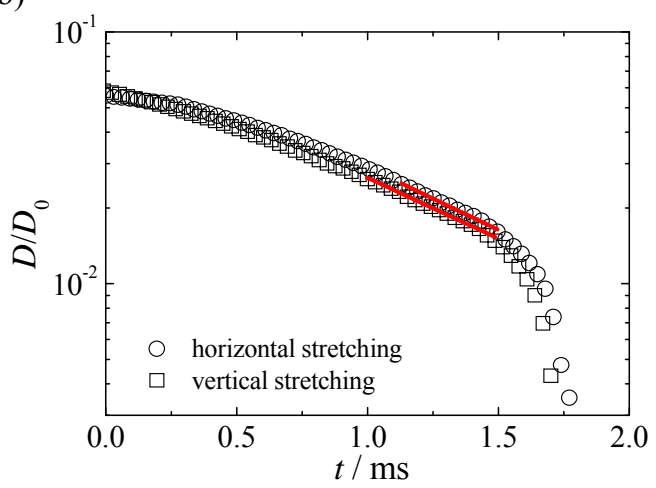

FIG. 2. Comparison between the time evolution of the minimum diameter of horizontally and vertically stretched filaments surrounded by air (a) and by silicone oil (b). The solid lines represent the fits to determine the extensional relaxation times. Blood samples were collected from donor D3. The curves were shifted in time to match at short times. 
Initially, the time evolution of the diameter of the filament surrounded by air and by oil is nearly independent of the stretching direction. After a certain time, which depends on the surrounding medium, it is possible to notice some difference between the diameter profiles measured in the horizontal and vertical positions. Nevertheless, the time evolution of the horizontal and vertical filaments in the time interval used to determine the extensional relaxation time is analogous and differences are of the order of the experimental error. Even though the difference is not significant, the remaining measurements were performed with vertical stretching of the blood samples to minimize the effect of cell sedimentation.

\section{B. Extensional relaxation time of human blood}

The diameter of the filament as a function of time is shown in Fig. 3 for three whole blood samples collected from different donors and also for human plasma. The filament diameter evolution for a Newtonian fluid and for two viscoelastic fluids based on polyacrylamide (PAA) used in Sousa et al. [20] is also shown for comparison purposes. The Newtonian fluid consists of 23.6 wt.\% glycerol in water, with a shear viscosity of $2 \mathrm{mPa} \cdot \mathrm{s}$ measured using a shear rheometer (Physica MCR301, Anton Paar) at $21^{\circ} \mathrm{C}$, which is similar to the value reported for the shear viscosity of plasma at the same temperature [32]. The viscoelastic fluids are aqueous solutions of PAA with weight concentrations of 2 and $5 \mathrm{ppm}$ [20]. The results obtained for whole blood and plasma samples collected from the same donor (D3) are compared, in addition to the results obtained from whole blood samples collected from donors D11 and D12, which have similar hematocrits but present some differences in terms of the time evolution of the blood filament. 


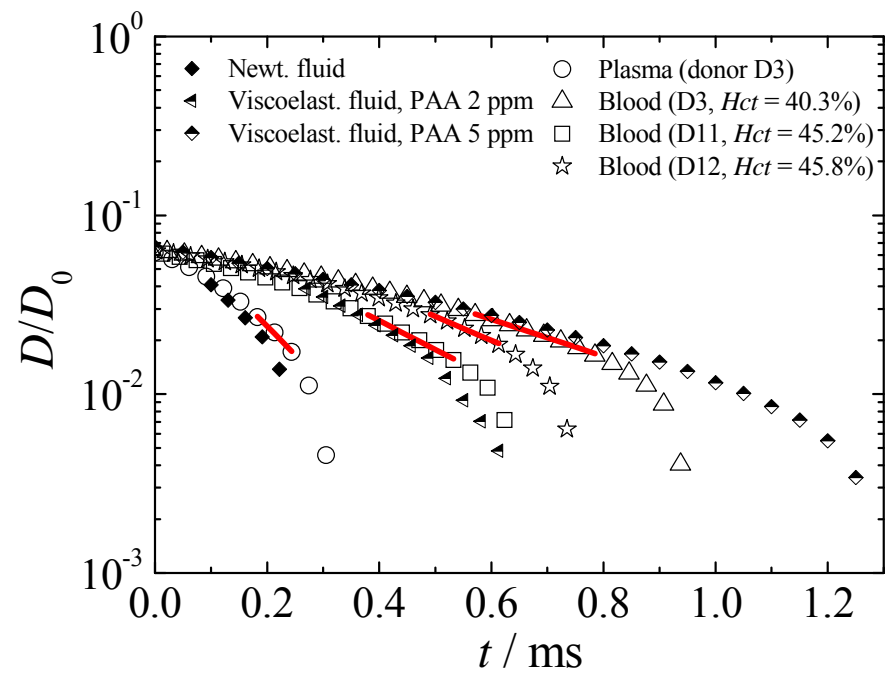

FIG. 3. Time evolution of the minimum filament diameter for whole human blood (donors D3, D11 and D12), human plasma (donor D3), a Newtonian fluid with a shear viscosity similar to that of plasma and two viscoelastic fluids with a different concentration of polymer ( $2 \mathrm{ppm}$ of PAA and $5 \mathrm{ppm}$ of PAA). All measurements were performed in air. Symbols represent experimental data and the lines represent the fit to determine the extensional relaxation times. The curves were shifted in time to match at short times.

Whole blood behaves as a Newtonian fluid during the major part of the thinning process, while the normal stresses are not yet large enough, with a dominance of the viscoelastic effect occurring towards the breakup when the filament diameter is less than about $60 \mu \mathrm{m}$. The time interval where the elasto-capillary regime was identified is also shown in Fig. 3 as solid lines. In this regime, the diameter of the filament decreases exponentially with time [30] and it is possible to determine the whole blood relaxation time (Eq. 1) from the slope of diameter as a function of time in semi-log coordinates. In order to select the relevant region of interest, we first perform a visual inspection of the filament to ensure a cylindrical shape in the time interval selected. Figure 4 shows a sequence of images taken during the filament thinning of a whole blood sample 
surrounded by air (Fig. 4a1) and by the silicone oil (Fig. 4a2) and also of a human plasma sample surrounded by air (Fig. 4b1) and by the silicone oil (Fig. 4b2).
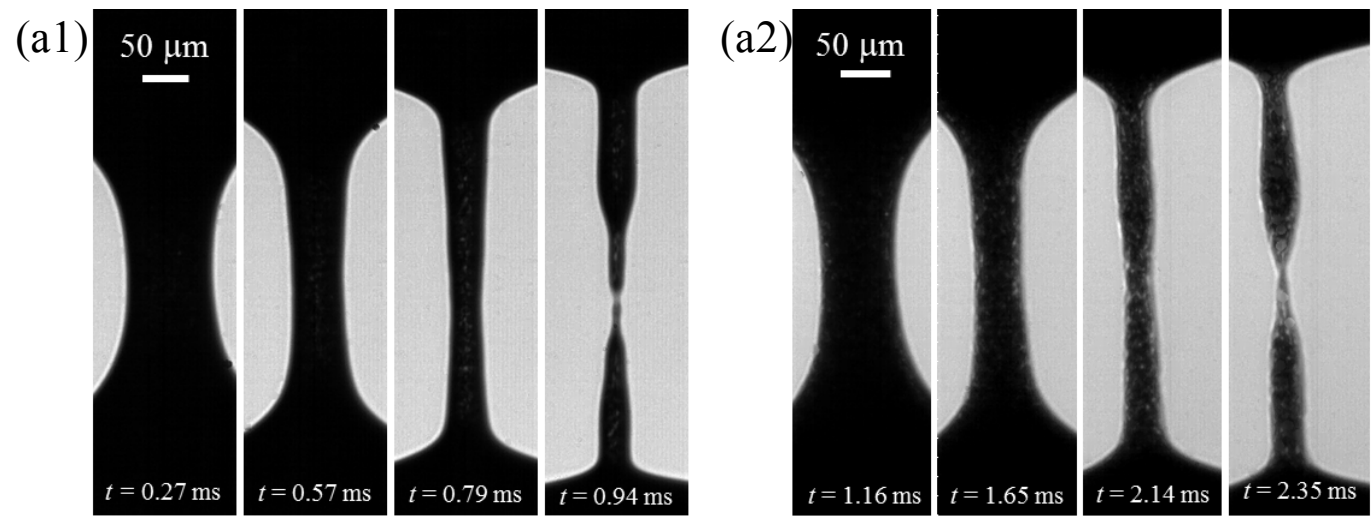

(b1)
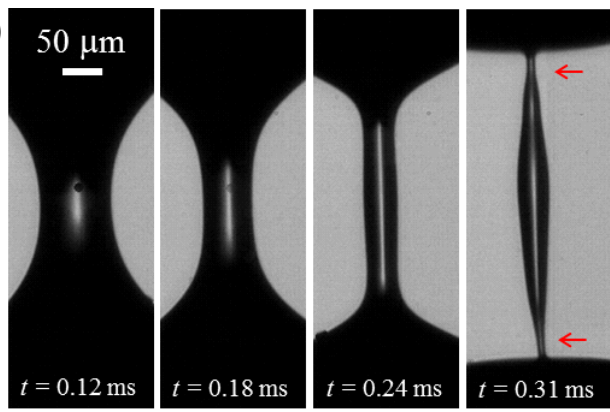

(b2)
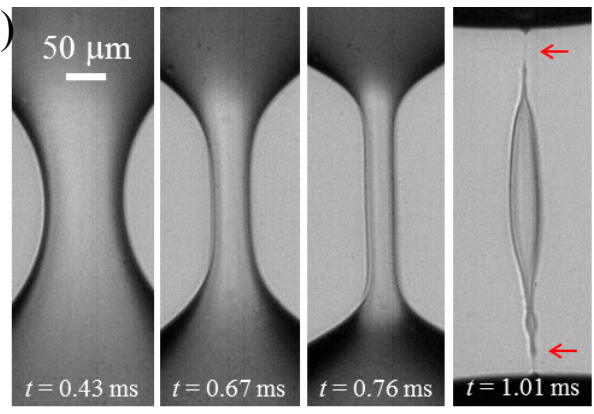

FIG. 4. Sequence of images showing the evolution of a filament of whole human blood (a1 and a2) and human plasma (b1 and b2) at four instants of time. Images (a1) and (b1) correspond to measurements in air, whereas images (a2) and (b2) correspond to measurements in the silicone oil. All blood and plasma samples shown were collected from donor D3.

For the whole blood sample, a quasi-cylindrical filament is observed in the time interval of $0.57 \leq t / \mathrm{ms}<0.79$ for filament in air and $1.65 \leq t / \mathrm{ms}<2.14$ for filament in oil, when the balance between viscoelasticity and capillarity governs the thinning of the thread. Near the final stage of the thinning process of whole blood $(t=0.94 \mathrm{~ms}$ in Fig. 4a1 and $t=2.35 \mathrm{~ms}$ in Fig. 4a2), the filament shows an irregular shape instead of a nearly cylindrical shape due to a non-uniform distribution of $\mathrm{RBC}$ along the filament, 
which is not considered in the quantification of the relaxation time. For the thinning in silicone oil, at $t=2.35 \mathrm{~ms}$ shown in Fig. 4a2, it is possible to observe a thick region with clusters of cells (dark part of the filament) as well as a plasma-rich zone (lighter part at the center of the filament), where the filament becomes thinner and eventually breaks. The region of the filament with a lower local RBC concentration exhibits a smaller effective viscosity and consequently the initial filament thinning is faster. Analogous observations were found in the thinning dynamics of particulate suspensions in a Newtonian matrix [31,33,34] and of concentrated cornstarch dispersions [35]. Additionally, we observed that the breakup typically occurs in the central region of the filament. When the filament exhibits an irregular shape, due to a non-uniform distribution of cells, the average diameter of the thread is typically less than $30 \mu \mathrm{m}$ and since the average diameter of erythrocytes and leukocytes is around $8 \mu \mathrm{m}$ and $15 \mu \mathrm{m}$, respectively, they occupy a significant portion of the filament.

It is important to note that the temporal evolution of the filament diameter of whole blood, shown in Fig. 3, is comparable to that observed for two highly diluted viscoelastic fluids, attesting the weak elastic character of whole blood. Sousa et al. [20] measured extensional relaxation times of approximately $90 \mu$ s and $180 \mu$ s for the 2 ppm and 5 ppm PAA solutions, respectively. These values of relaxation time are similar to those estimated for the extensional relaxation time of whole blood, as presented below. In addition, as can be seen in Fig. 3, the diameter profiles of whole blood samples obtained from different donors present some differences as expected due to donor to donor variability, particularly in terms of hematocrit and concentration of proteins. As expected, the capillary bridge of the human plasma breaks faster than that for whole blood (cf. Fig. 3), since the presence of the cells increases the bulk viscosity and consequently delays the initial thinning process and the time to breakup. For the plasma 
sample, the temporal evolution of the filament diameter shows a small elasto-capillary region $(0.18<t / \mathrm{ms} \leq 0.24$ in air and $0.67<t / \mathrm{ms} \leq 0.76$ in oil), in which the filament presents a quasi-cylindrical shape (cf. Fig. $4 \mathrm{~b} 1$ and $4 \mathrm{~b} 2$ ) and the extensional relaxation time can be estimated. Near breakup ( $t \geq 0.28 \mathrm{~ms}$ in air and $t \geq 0.95 \mathrm{~ms}$ in silicone oil), the filament at the top and bottom ends thins faster than at the central region, which eventually leads to the formation of a single central droplet. Although the breakup of the plasma filament looks similar to that of a Newtonian fluid, the thin tails close to the top and bottom plates, indicated by the arrow in Figure $4 \mathrm{~b} 1$ and $4 \mathrm{~b} 2$, suggest a slight viscoelastic behavior of human plasma. A similar filament shape was observed in the filament thinning and breakup of weakly elastic fluids, in which the formation of a tail indicates that viscoelasticity acts against the breakup by increasing the breakup time $[36,37]$. Nevertheless, the very weak viscoelastic character of human plasma seen here contrasts with previous observations of Brust et al. [19]. The differences in the human plasma behavior in extensional flow between these two works may perhaps be attributed to the different experimental conditions used in both investigations, such as the concentration of anticoagulant used in blood collection.

As can be seen in Fig. 3, the differences between the curves obtained for the Newtonian fluid and plasma are tenuous, being of the order of the experimental error. However, it is imperative to refer that the viscoelastic character of human plasma [19] and consequently of human blood is due to the presence of the cells and of other solutes, such as proteins. Moreover, it is important to highlight that the results obtained for such low-viscosity and low-elasticity fluids are close to the minimum measurable limit of the technique used. It is worth mentioning that we also measured the filament diameter of a plasma sample, which was obtained by allowing the blood cells to settle down by 
gravity for approximately $2 \mathrm{~h}$. Similar evolutions of the filament diameter were observed by using the plasma samples obtained by sedimentation and by centrifugation.

The extensional relaxation times of all blood samples were measured with the capillary bridge surrounded by air and also by the silicone oil bath. In addition to allowing the visualization of the cells inside the fluid filament, the use of the oil bath can prevent evaporation and the formation of a plasma protein surface layer at the liquid-air interface. Copley and King [38] showed that viscoelastic effects found in plasma are attributed to the surface layer plasma protein at the liquid-air interface. More recently, Jaishankar et al. [39] found that viscoelastic effects of bovine serum albumin solutions in shear flows arise from the presence of a viscoelastic layer, which results from the adsorption of protein also at the liquid-air interface. In this regard, Brust et al. [19] also performed measurements of the extensional rheology of plasma with capillary bridges surrounded by silicone oil in order to ensure that the viscoelastic behavior found for human plasma are not due to the viscoelastic layer formed by the adsorption of protein molecules at the air-liquid interface.

A comparison of the extensional relaxation times measured in air and in oil bath for all donors is presented in Fig. 5a. Figure 5b shows the extensional relaxation times measured in air and in the silicone oil as a function of the hematocrit for both male and female donors. The average values of the extensional relaxation times and the estimated standard deviation are listed in Table II. 
(a)

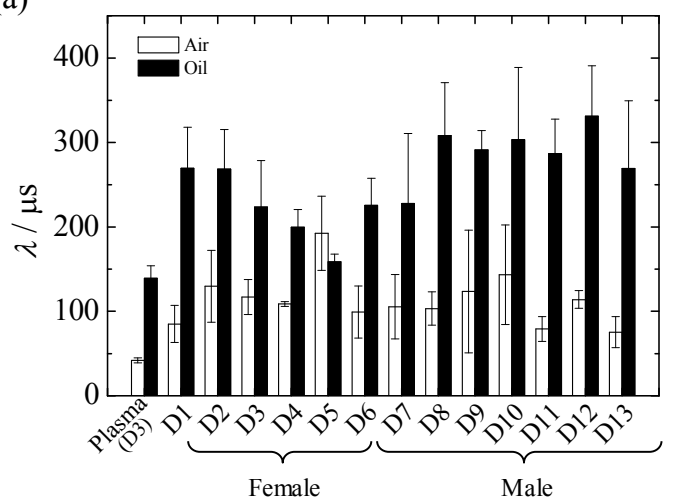

(b)

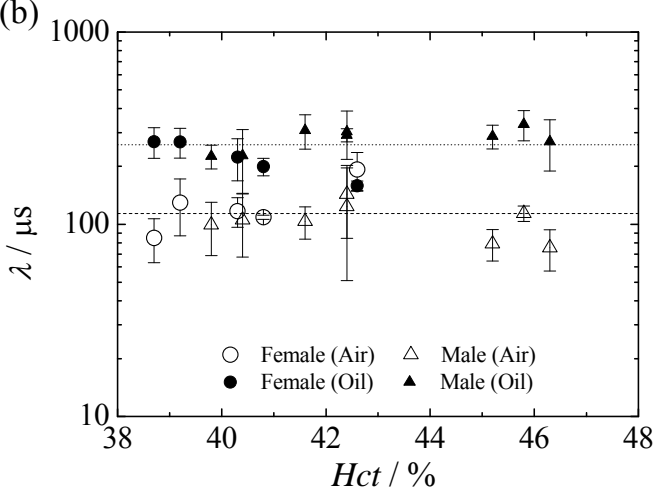

FIG. 5. (a) Extensional relaxation times measured with the whole blood filament surrounded by air and silicone oil for all donors. The extensional relaxation time measured for plasma (donor D3) is also shown. (b) Extensional relaxation time as a function of the hematocrit. The dashed and dotted lines represent the averages measured with the filament in air and in silicone oil, respectively.

TABLE II. Average values of the extensional relaxation times estimated for whole human blood and human plasma. The relaxation times were measured using the samples surrounded by air and by silicone oil.

\begin{tabular}{cccc}
\hline & & $\lambda_{\text {air }}(\mu \mathrm{s})$ & $\lambda_{\text {oil }}(\mu \mathrm{s})$ \\
\hline \multirow{2}{*}{$\frac{1}{0} \frac{1}{0}$} & Female & $127 \pm 36$ & $224 \pm 42$ \\
& Male & $105 \pm 21$ & $280 \pm 35$ \\
& Total & $114 \pm 30$ & $259 \pm 47$ \\
& & & \\
& Plasma (D3) & $42 \pm 3$ & $139 \pm 15$ \\
\hline \hline
\end{tabular}

The results obtained for whole blood filaments surrounded by air and by oil show that there is no discernible differences between the extensional relaxation time measured for female and male donors. The average value of $\lambda$ obtained when an outer liquid bath of low viscosity silicone oil is used is roughly twice the value of $\lambda$ obtained for filaments surrounded by air, in accordance to previous findings for polymer solutions [20]. For 
filaments surrounded by air, evaporation of water from the sample can lead to a faster decrease of the filament diameter in the elasto-capillary regime and consequently to a lower relaxation time [20]. Moreover, interfacial phenomena can also occur when the sample is surrounded by air, leading to a reduction of the relaxation time.

For human plasma, and in spite of its small elasticity, we also estimate the extensional relaxation time, which is approximately half the value for whole blood, demonstrating the importance of the red blood cells on the extensional behavior of whole blood.

In summary, the results suggest that the variation of the extensional relaxation time is not significant for the range of Hct investigated, which corresponds to healthy humans of both genders. Additionally, it is interesting to note that for an isolated RBC, the relaxation time reported in the literature, measured by optical tweezers, and micropipette aspiration among other techniques, is of the order of $0.17 \pm 0.08 \mathrm{~s}$ [40]. The $\mathrm{RBC}$ relaxation time, defined as the time that a deformed $\mathrm{RBC}$ requires to recover its original biconcave shape, is determined as the ratio of the elastic shear modulus and the membrane surface viscosity [40]. However, the significant difference between the relaxation time reported for a single $\mathrm{RBC}$ and the value found in this investigation for whole blood is due to the nature of the deformations involved in the measurements. For a single $\mathrm{RBC}$, the characteristic relaxation time is based on $\mathrm{RBC}$ shear properties, whereas here the measurement of the extensional relaxation time is performed in a pure extensional flow.

In order to better visualize the movement of $\mathrm{RBC}$ in the filament, we performed experiments in the oil bath using two fluids with a lower concentration of RBC. One fluid is composed of $20 \%(\mathrm{v} / \mathrm{v})$ of erythrocytes in human plasma and the other fluid has the same concentration of cells but dispersed in PBS. Figure 6a shows the time 
evolution of the minimum filament diameter of such fluids as well as for whole blood, plasma and for pure PBS.

As expected, there is a significant effect of the hematocrit on the thinning process since a higher concentration of cells leads to an increased resistance during the filament thinning, which delay the breakup. Moreover, for the same Hct, it is possible to observe a clear difference between the thinning obtained for the RBC in plasma and in PBS. In fact, the capillary thinning of the PBS based RBC sample shows Newtonian characteristics, whereas the tensile stress due to the viscoelastic character in the capillary thinning of the plasma-based sample delays the time to breakup. The presence of proteins in the plasma promotes the viscoelastic character of this physiological fluid. In fact, the concentration of proteins may lead to variations of whole blood rheology $[41,42]$.

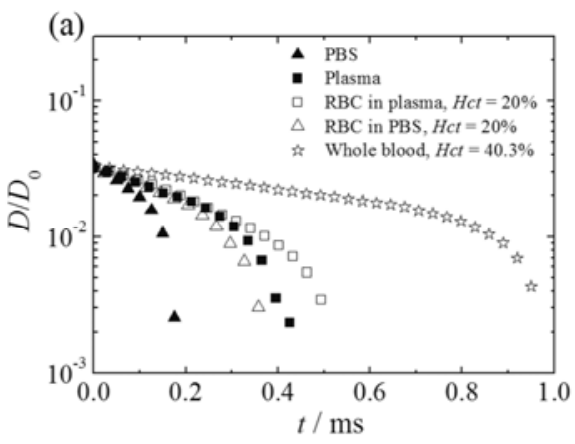

(b)

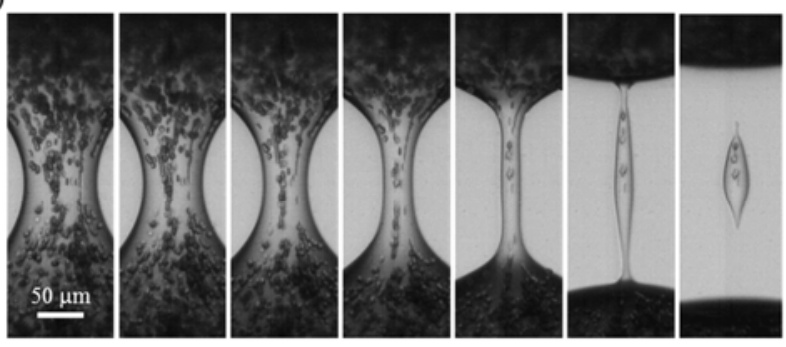

FIG. 6. (a) Comparison of the minimum filament diameter as a function of time for PBS, human plasma, a solution of $20 \%(\mathrm{v} / \mathrm{v})$ of RBC in plasma, a solution of $20 \%(\mathrm{v} / \mathrm{v})$ of RBC in PBS and whole human blood (all surrounded by silicone oil). All blood samples were collected from donor D3. The curves were shifted in time to match at short times. (b) Sequence of images for the fluid composed of $20 \%(\mathrm{v} / \mathrm{v})$ of RBC in plasma. The experiment was conducted with the fluid immersed in the silicone oil in order to reduce light refraction, and the time interval between each image is $122 \mu \mathrm{s}$. 
The time evolution of the filament thinning and breakup of $20 \%(\mathrm{v} / \mathrm{v}) \mathrm{RBC}$ in plasma is presented in Fig. 6b. It is possible to observe a small level of RBC aggregation in the samples of RBC in plasma (cf. Fig. 6b), whereas the samples of RBC in PBS do not reveal significant aggregation. The presence of these $\mathrm{RBC}$ aggregates may also be a reason for the modest increase of the time to breakup of $\mathrm{RBC}$ in plasma. As discussed previously, a heterogeneous concentration of cells is detected in the filament, especially near the breakup. Moreover, during the thinning process, and even though most cells are progressively advected out of the filament and reach the end drops, as also observed by McIlroy and Harlen [34] for a particulate suspension, some cells remain in the central part of the filament forming an elongated droplet. The higher viscosity of the particlerich zone means that it tends not to thin further. Even though the deformability of RBC would suggest their deformation during the capillary thinning, such behavior was not clearly visible in our experiments most probably because the typical time required by the $\mathrm{RBC}$ to deform is higher than the required time for the filament to thin and breakup.

\section{Effect of anticoagulant and effect of sample aging}

In order to test the effect of the anticoagulant on the evolution of the filament of whole blood, and consequently on the measured relaxation time, we also performed experiments with a blood sample without adding anticoagulant. Blood was collected directly to a disposable plastic syringe and a small sample of blood was transferred to the measuring device in order to form the liquid bridge in the shortest possible time (approximately within $1 \mathrm{~min}$ ). Figure 7 shows the variation of the filament diameter with time for two consecutive measurements performed using blood taken in the same withdrawal as well as for a blood sample with anticoagulant. Images (a) - (d) presented in Fig. 7 correspond to four instants in the first measurement and images (e) - (h) 
correspond to four instants in the second measurement, in both cases using the blood sample without anticoagulant. All the events are indicated in the plot of Fig. 7.
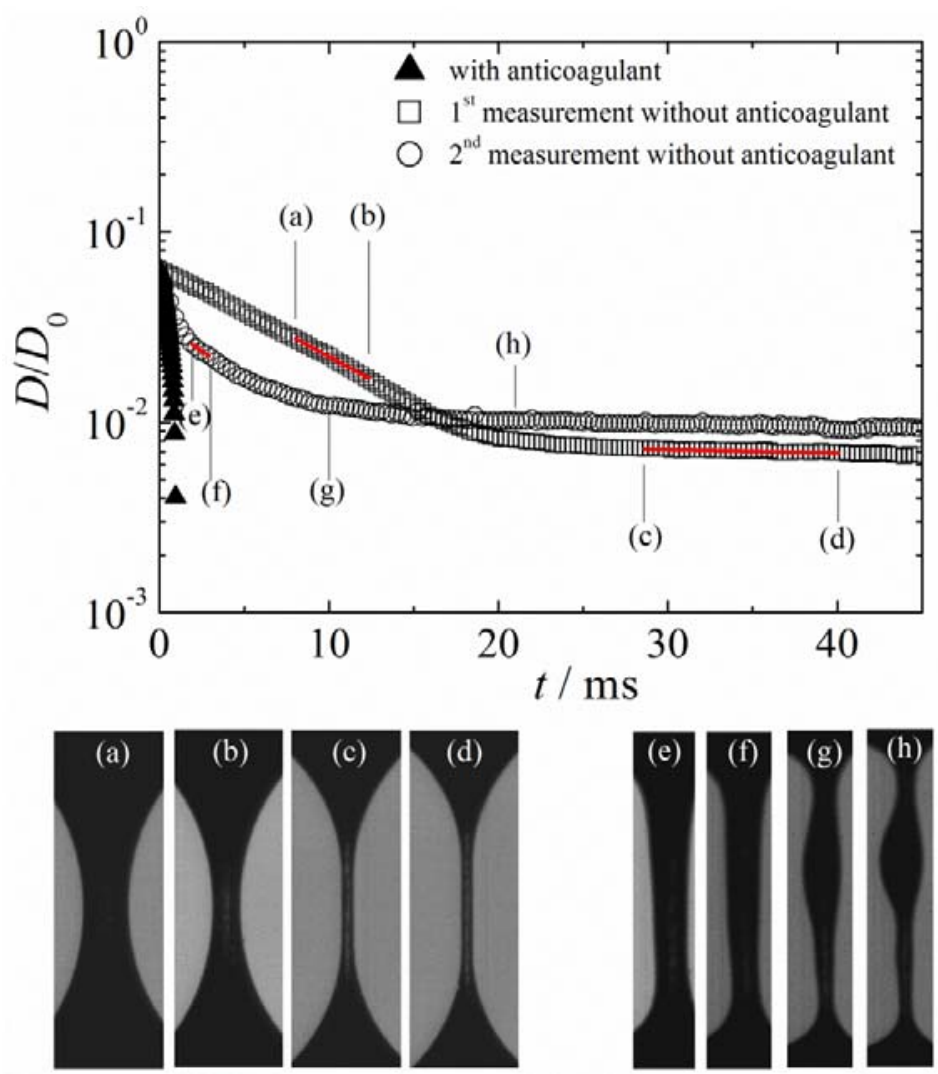

FIG. 7. Time evolution of the minimum filament diameter of whole blood sample with (solid symbols) and without anticoagulant (hollow symbols). Images (a)-(d) correspond to the first measurement without anticoagulant, whereas images (e)-(h) correspond to the second measurement performed with the whole blood sample without anticoagulant. Both blood samples with and without anticoagulant were collected from the same donor (D3). The red solid lines in the plot represent the fit to determine the extensional relaxation times. All measurements were performed in air.

For both measurements performed without anticoagulant, the filament thinning takes longer than for the blood sample with anticoagulant and the breakup did not occur in the course of the experiment. In the first measurement, the time evolution of the filament 
diameter shows two distinct quasi-linear regions: one for $t \leq 17 \mathrm{~ms}$, and the other for $t \gtrsim 17 \mathrm{~ms}$. The extensional relaxation time determined in the time interval between (a) and (b) is $\lambda=3 \mathrm{~ms}$, whereas that for the interval between (c) and (d) is $\lambda=83 \mathrm{~ms}$. It is important to note that for $t \geq 25 \mathrm{~ms}$, the variation of the filament diameter is very small $(\sim 1.5 \mu \mathrm{m})$. At this stage, the surface-to-volume ratio increases significantly leading to the formation of a rigid structure in the filament, possibly due to coagulation and to an increase in mass transfer between the filament and the surroundings air (filament drying). Hence, a comparison between the relaxation times for both regions suggests that coagulation of blood leads to a significant increase of the extensional relaxation time.

In the subsequent measurement with blood collected in the same withdrawal, and carried out approximately 6 min after blood collection, the loaded blood sample showed an extensional relaxation time of $\lambda=2 \mathrm{~ms}$, estimated from the time interval between points (e) and (f). In this second experiment, we verified that for $t \geq 6.5 \mathrm{~ms}$, a bead is formed in the filament and its shape remains nearly unchanged up to the end of the experiment, perhaps due to clot formation in that region.

In summary, the absence of anticoagulant increases the value of the extensional relaxation time by about one order of magnitude, to a value which is close to the values reported by Brust et al. [19]. These results can reveal that the discrepancies between the extensional relaxation time of whole human blood found in the two works can be explained by the differences in the protocols used.

It is important to mention that even in some experiments using blood samples with anticoagulant a thin filament remained for a long period at the final stage of the thinning process. This phenomenon seems to occur randomly and may be related with the 
formation of a protein surface layer at the air-blood interface [39], which in this case leads just to a modest increase (around 30\%) of the value of the measured relaxation time.

In order to assess the effect of aging, we use again the regular collection procedure and a blood sample was adequately stored at $4^{\circ} \mathrm{C}$ in a test tube containing anticoagulant ( $\left.\mathrm{K}_{3} \mathrm{EDTA}\right)$ for a period of $24 \mathrm{~h}$. Figure 8a compares the time evolution of filament diameter for whole blood and plasma samples measured just after blood withdrawal and $24 \mathrm{~h}$ later. In order to obtain the $24 \mathrm{~h}$ old plasma sample, a tube containing the whole blood sample was allowed to stand at $4^{\circ} \mathrm{C}$ for a period of $24 \mathrm{~h}$ so that blood cells could settle down at the bottom of the tube due to gravity and the upper liquid layer in the tube, corresponding to plasma, was carefully removed and used in the experiments. In Fig. 8a we also show the diameter profile obtained using the reference Newtonian fluid. In these experiments, all filaments are surrounded by air.

Aging of the whole blood sample and of the plasma sample affects the diameter profiles and consequently the extensional relaxation times. In fact, the breakup time of the filaments, for the $24 \mathrm{~h}$ old blood sample and for the $24 \mathrm{~h}$ old plasma sample, decreases as well as the measured value of the extensional relaxation time. The same observations were obtained from measurements in which the filament is surrounded by oil (not shown here). It is possible that RBC experience changes in their rheological and mechanical properties with time [43] when, outside the circulatory system as a consequence of interfacial phenomena, resulting in hardening of $\mathrm{RBC}$, and consequently decreasing their capability to deform. 


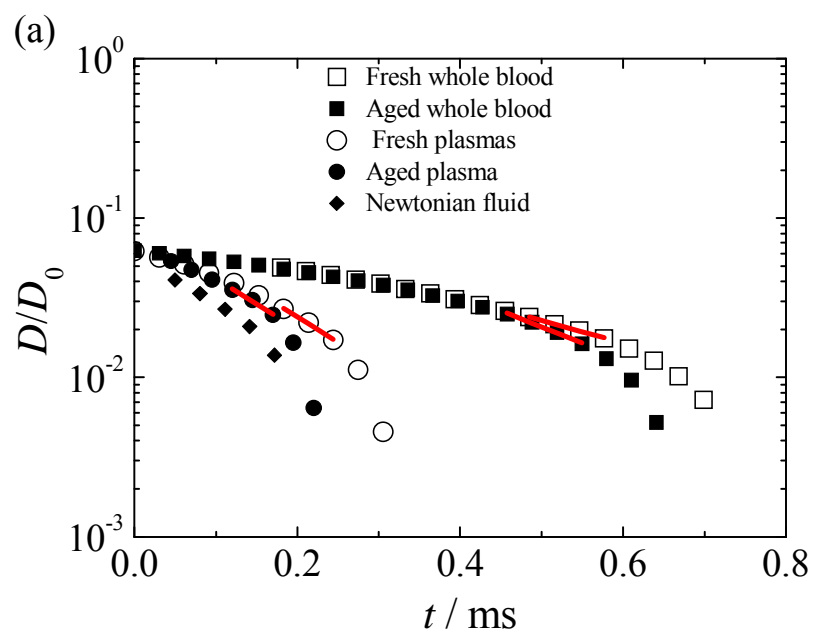

(b)

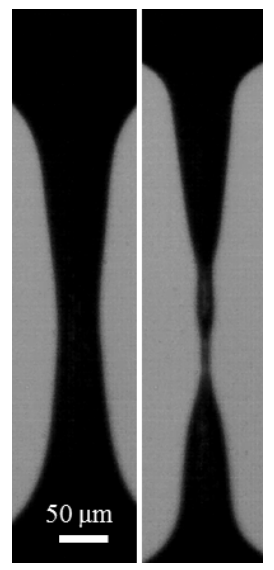

(c)

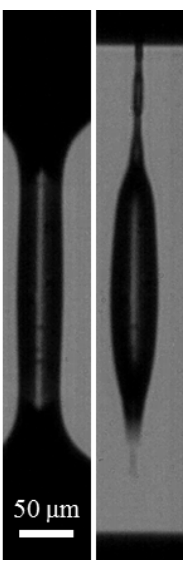

(d)

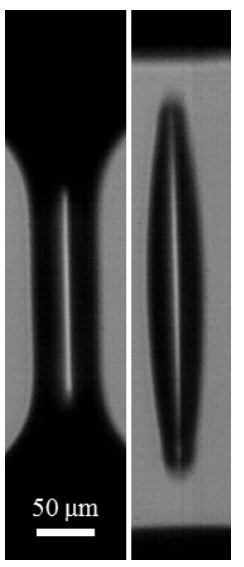

FIG. 8. (a) Variation with time of the minimum filament diameter for whole blood, human plasma and a Newtonian fluid (aqueous solution of $23.6 \mathrm{wt} \%$ glycerol). The hollow symbols represent the results obtained with fresh samples and the solid symbols represent the results obtained with $24 \mathrm{~h}$ old samples. The solid lines represent the fits used to determine the extensional relaxation times. The curves were shifted in time to match at short times. Whole blood samples were collected from donor D9 and human plasma was collected from donor D3. Photographs of the filament breakup for: (b) a $24 \mathrm{~h}$ old whole blood sample (time between the two frames is $153 \mu \mathrm{s}$ ); (c) a $24 \mathrm{~h}$ old plasma sample (time between the two frames is $75 \mu \mathrm{s}$ ); (d) a Newtonian fluid (time between the two frames is $61 \mu \mathrm{s})$. All measurements were performed with the filament surrounded by air. 
In each of Fig. $8 b, 8 c$ and $8 d$ we show two representative images of the filaments during the thinning process for a $24 \mathrm{~h}$ old blood sample, a $24 \mathrm{~h}$ old plasma sample and for the Newtonian fluid, respectively.

As discussed in Section III. B, the breakup of the whole blood filament occurs in a plasma-rich zone due to the variation of the $\mathrm{RBC}$ concentration. In contrast, the breakup of the plasma bridge occurs at the top and bottom of the filament, where a thin fluid tail is formed as illustrated in Fig. 8c, which also differs from the Newtonian counterpart (Fig. 8d).

\section{Conclusions}

Capillary breakup experiments were used to investigate the elongational flow of whole human blood. In addition, the temporal evolution of human plasma and of a Newtonian fluid, with a shear viscosity similar to that of plasma, were also investigated. Due to the small relaxation time of blood in uniaxial extensional flow, we combined the slow retraction method with high-speed imaging techniques and a sub-pixel technique for the measurement of the filament diameter evolution with time. The thinning dynamics of all samples were investigated for capillary bridges surrounded by air and by an immiscible low viscosity silicone oil. The use of the immiscible oil reduces liquid loss by evaporation and allows the visualization of blood cells inside the filament, while also avoiding the formation of a protein surface layer at the air-water interface as reported by Jaishankar et al. [39]. For the range of hematocrit levels investigated, the average extensional relaxation times measured in air and in oil are $114 \pm 30 \mu \mathrm{s}$ and $259 \pm 47 \mu \mathrm{s}$, respectively. The extensional relaxation time found for human plasma is approximately half of the value for whole human blood. Moreover, the capillary thinning of RBC in 
plasma shows viscoelastic characteristics, oppositely to RBC in PBS, due to the presence of proteins in the plasma. Hence, it would be important to investigate in future works the relation between protein concentration and the extensional relaxation time of human plasma, as well as the effect of protein concentration on the formation of RBC aggregates and on the extensional relaxation time of whole blood.

For the conditions investigated and the protocol employed in this work, we concluded that whole blood behaves as a slight viscoelastic fluid in extensional flow, with some similarities to the behavior of a particulate suspension during the filament thinning. In particular, near the filament breakup, the cell concentration becomes heterogeneous and the plasma-rich zones thin faster due to the decrease in local apparent viscosity. Moreover, the results show that aging of whole blood reduces the extensional relaxation time and therefore this should be considered when planning protocols for future investigations.

\section{Acknowledgements}

P. C. Sousa thanks Fundação para a Ciência e a Tecnologia (FCT) for the financial support through POPH/FSE and national funding from MEC (fellowship SFRH/BPD/75258/2010). The help of the nursing staff at Hospital de S. João (Porto, Portugal), especially nurse Rosa Silva, in the collection of blood samples is acknowledged. 


\section{References}

[1] Puig-De-Morales-Marinkovic, M., K. T. Turner, J. P. Butler, J. J. Fredberg, and S. Suresh, "Viscoelasticity of the human red blood cell," Am J Physiol-Cell Ph 293, C597-C605 (2007).

[2] Li, X. J., Z. L. Peng, H. Lei, M. Dao, and G. E. Karniadakis, "Probing red blood cell mechanics, rheology and dynamics with a two-component multi-scale model," Philos T R Soc A 372, 20130389 (2014).

[3] Thurston, G. B., "Viscoelasticity of Human Blood," Biophys J 12, 1205-1217 (1972).

[4] Sousa, P. C., F. T. Pinho, M. A. Alves, and M. S. N. Oliveira, "A review of hemorheology: Measuring techniques and recent advances," Korea-Aust Rheol J 28, 1-22 (2016).

[5] Thurston, G. B. and N. M. Henderson, "Viscoelasticity of human blood," Handbook of Hemorheology and Hemodynamics, O.K. Barskurt, eds. (Amsterdam; Washington DC, 2007), pp. 72-90.

[6] Vlastos, G., D. Lerche, B. Koch, O. Samba, and M. Pohl, "The effect of parallel combined steady and oscillatory shear flows on blood and polymer solutions," Rheol Acta 36, 160-172 (1997).

[7] Sousa, P. C., J. Carneiro, R. Vaz, A. Cerejo, F. T. Pinho, M. A. Alves, and M. S. N. Oliveira, "Shear viscosity and nonlinear behavior of whole blood under large amplitude oscillatory shear," Biorheology 50, 269-282 (2013).

[8] Campo-Deaño, L., R. P. A. Dullens, D. G. A. L. Aarts, F. T. Pinho, and M. S. N. Oliveira, "Viscoelasticity of blood and viscoelastic blood analogues for use in polydymethylsiloxane in vitro models of the circulatory system," Biomicrofluidics 7, 034102 (2013). 
[9] Lee, S. S., Y. Yim, K. H. Ahn, and S. J. Lee, "Extensional flow-based assessment of red blood cell deformability using hyperbolic converging microchannel," Biomed Microdevices 11, 1021-1027 (2009).

[10] Sousa, P. C., F. T. Pinho, M. S. N. Oliveira, and M. A. Alves, "Extensional flow of blood analog solutions in microfluidic devices," Biomicrofluidics 5, 014108 (2011).

[11] Yaginuma, T., M. S. N. Oliveira, R. Lima, T. Ishikawa, and T. Yamaguchi, "Human red blood cell behavior under homogeneous extensional flow in a hyperbolic-shaped microchannel," Biomicrofluidics 7, 054110 (2013).

[12] Cha, S., T. Shin, S. S. Lee, W. Shim, G. Lee, S. J. Lee, Y. Kim, and J. M. Kim, "Cell Stretching Measurement Utilizing Viscoelastic Particle Focusing," Anal Chem 84, 10471-10477 (2012).

[13] Shaqfeh, E. S. G., "The dynamics of single-molecule DNA in flow," J NonNewton Fluid 130, 1-28 (2005).

[14] Perkins, T. T., D. E. Smith, and S. Chu, "Single polymer dynamics in an elongational flow," Science 276, 2016-2021 (1997).

[15] Down, L. A., D. V. Papavassiliou, and E. A. O'Rear, "Significance of Extensional Stresses to Red Blood Cell Lysis in a Shearing Flow," Ann Biomed Eng 39, 1632-1642 (2011).

[16] Arratia, P. E., C. C. Thomas, J. Diorio, and J. P. Gollub, "Elastic instabilities of polymer solutions in cross-channel flow," Phys Rev Lett 96, 144502 (2006).

[17] Oliveira, M. S. N., F. T. Pinho, R. J. Poole, P. J. Oliveira, and M. A. Alves, "Purely elastic flow asymmetries in flow-focusing devices," J Non-Newton Fluid 160, 31-39 (2009). 
[18] Haward, S. J., M. S. N. Oliveira, M. A. Alves, and G. H. McKinley, "Optimized Cross-Slot Flow Geometry for Microfluidic Extensional Rheometry," Phys Rev Lett 109, 128301 (2012).

[19] Brust, M., C. Schaefer, R. Doerr, L. Pan, M. Garcia, P. E. Arratia, and C. Wagner, "Rheology of Human Blood Plasma: Viscoelastic Versus Newtonian Behavior," Phys Rev Lett 110, 078305 (2013).

[20] Sousa, P. C., E. J. Vega, R. G. Sousa, J. M. Montanero, and M. A. Alves, "Measurement of relaxation times in extensional flow of weakly viscoelastic polymer solutions," Rheol Acta 56, 11-20 (2017).

[21] Baskurt, O. K., M. Boynard, G. C. Cokelet, P. Connes, B. M. Cooke, S. Forconi, F. L. Liao, M. R. Hardeman, F. Jung, H. J. Meiselman, G. Nash, N. Nemeth, B. Neu, B. Y. Sandhagen, S. Shin, G. Thurston, and J. L. Wautier, "New guidelines for hemorheological laboratory techniques," Clin Hemorheol Micro 42, 75-97 (2009).

[22] Campo-Deaño, L. and C. Clasen, "The slow retraction method (SRM) for the determination of ultra-short relaxation times in capillary breakup extensional rheometry experiments," J Non-Newton Fluid 165, 1688-1699 (2010).

[23] Vega, E. J., J. M. Montanero, and J. Fernandez, "On the precision of optical imaging to study free surface dynamics at high frame rates," Exp Fluids 47, 251261 (2009).

[24] Goldin, M., J. Yerushal., R. Pfeffer, and R. Shinnar, "Breakup of a Laminar Capillary Jet of a Viscoelastic Fluid," J Fluid Mech 38, 689-711 (1969).

[25] Bazilevsky, A. V., V. M. Entov, and A. N. Rozhkov, 1990, "Liquid filament microrheometer and some of its applications," Proc. Third European Rheology Conference, D.R. Oliver, Elsevier, pp. 41-43. 
[26] Anna, S. L. and G. H. McKinley, "Elasto-capillary thinning and breakup of model elastic liquids," J Rheol 45, 115-138 (2001).

[27] Clasen, C., "Capillary breakup extensional rheometry of semi-dilute polymer solutions," Korea-Aust Rheol J 22, 331-338 (2010).

[28] Rodd, L. E., T. P. Scott, J. J. Cooper-White, and G. H. McKinley, "Capillary break-up rheometry of low-viscosity elastic fluids," Appl Rheol 15, 12-27 (2005).

[29] Oliveira, M. S. N. and G. H. McKinley, "Iterated stretching and multiple beadson-a-string phenomena in dilute solutions of highly extensible flexible polymers," Phys Fluids 17, 071704 (2005).

[30] Entov, V. M. and E. J. Hinch, "Effect of a spectrum of relaxation times on the capillary thinning of a filament of elastic liquid," J Non-Newton Fluid 72, 31-53 (1997).

[31] Mathues, W., C. McIlroy, O. G. Harlen, and C. Clasen, "Capillary breakup of suspensions near pinch-off," Phys Fluids 27, 093301 (2015).

[32] Thurston, G. B., "Viscoelastic Properties of Blood and Blood Analogs," Advances in hemodynamics and hemorheology, T.V. How, eds. (JAI Press LTD, London, 1996), pp. 1-30.

[33] Alexandrou, A. N., A. V. Bazilevskii, V. M. Entov, A. N. Rozhkov, and A. Sharaf, "Breakup of a Capillary Bridge of Suspensions," Fluid Dynam 45, 952$964(2010)$.

[34] McIlroy, C. and O. G. Harlen, "Modelling capillary break-up of particulate suspensions," Phys Fluids 26, 033101 (2014). 
[35] Roché, M., H. Kellay, and H. A. Stone, "Heterogeneity and the Role of Normal Stresses during the Extensional Thinning of Non-Brownian Shear-Thickening Fluids," Phys Rev Lett 107, 134503 (2011).

[36] Vadillo, D. C., W. Mathues, and C. Clasen, "Microsecond relaxation processes in shear and extensional flows of weakly elastic polymer solutions," Rheol Acta 51, 755-769 (2012).

[37] Vadillo, D. C., T. R. Tuladhar, A. C. Mulji, S. Jung, S. D. Hoath, and M. R. Mackley, "Evaluation of the inkjet fluid's performance using the "Cambridge Trimaster" filament stretch and break-up device," J Rheol 54, 261-282 (2010).

[38] Copley, A. L. and R. G. King, "Viscous resistance of thromboid (thrombus-like) surface layers in systems of plasma proteins including fibrinogen," Thrombosis Research 1, 1-17 (1972).

[39] Jaishankar, A., V. Sharma, and G. H. McKinley, "Interfacial viscoelasticity, yielding and creep ringing of globular protein-surfactant mixtures," Soft Matter 7, 7623-7634 (2011).

[40] Tomaiuolo, G., "Biomechanical properties of red blood cells in health and disease towards microfluidics," Biomicrofluidics 8, 051501-19 (2014).

[41] Brust, M., O. Aouane, M. Thiebaud, D. Flormann, C. Verdier, L. Kaestner, M. W. Laschke, H. Selmi, A. Benyoussef, T. Podgorski, G. Coupier, C. Misbah, and C. Wagner, "The plasma protein fibrinogen stabilizes clusters of red blood cells in microcapillary flows," Sci Rep-Uk 4, 4348 (2014).

[42] Flormann, D., K. Schirra, T. Podgorski, and C. Wagner, "On the rheology of red blood cell suspensions with different amounts of dextran: separating the effect of aggregation and increase in viscosity of the suspending phase," Rheol Acta 55, 477-483 (2016). 
[43] Wang, Y., G. X. You, P. P. Chen, J. J. Li, G. Chen, B. Wang, P. L. Li, D. Han, H. Zhou, and L. Zhao, "The mechanical properties of stored red blood cells measured by a convenient microfluidic approach combining with mathematic model," Biomicrofluidics 10, 024104-14 (2016). 